PRAXIS

ecucativa

Universidad Nacional de La Pampa

Facultad de Ciencias Humanas

Instituto de Ciencias de la Educación

para la investigación interdisciplinaria

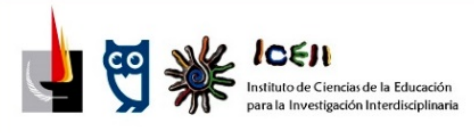

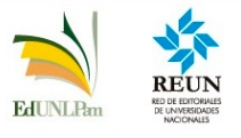

ISSN 2313-934X

SANTA ROSA, LA PAMPA, ARGENTINA

Correo electrónico: iceii@humanas.unlpam.edu.ar

Disponible en https://cerac.unlpam.edu.ar/index.php/praxis

La diferenciación de la fuerza de trabajo y las características socioeconómicas del alumnado de la educación secundaria de personas jóvenes y adultas en Argentina en los años recientes. Artículo de Julián Olivares. Praxis educativa, Vol. 26, No 1 enero - abril 2022. E ISSN 2313-934X. pp. 1-20. https://dx.doi.org/10.19137/praxiseducativa-2022-260113

Esta obra se publica bajo Licencia Creative Commons 4.0 Internacional CC BY-NC-SA Atribución, No Comercial, Compartir igual

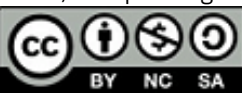

\title{
La diferenciación de la fuerza de trabajo y las características socioeconómicas del alumnado de la educación secundaria de personas jóvenes y adultas en Argentina en los años recientes
}

Differentiation of the workforce and the socioeconomic characteristics of the student body of thesecondary education for young and adult persons in Argentina in recent years

A diferenciação da força de trabalho e as característica socioeconômicas do corpo estudantil do ensino médio de pessoas jovens e adultas na Argentina nos últimos anos

\section{Julián Olivares}

Universidad Nacional de General Sarmiento. Consejo Nacional de Ciencia y Tecnología, CONICET, Argentina olivares.julian.90@gmail.com 


\title{
Resumen
}

Este trabajo tiene como objetivo analizar en qué medida la creciente diferenciación de la fuerza de trabajo afecta al alumnado de la educación secundaria de personas jóvenes y adultas en Argentina en los años recientes. Para ello, se utilizan distintos relevamientos realizados por el INDEC para llevar adelante una aproximación estadística acerca de las características socioeconómicas del estudiantado del secundario de la EDJA en todo el país, buscando determinar cuál es el grado en que sus condiciones de reproducción están mediadas por situaciones tales como la desocupación, el empleo precario y la pobreza, entre otros aspectos. Los resultados muestran que dichos alumnos y alumnas pertenecen, en general, a sectores muy afectados por la creciente diferenciación de la fuerza laboral, lo cual se manifiesta en que tienen condiciones de vida más deterioradas que el promedio de la población adulta.

Palabras clave: educación secundaria, educación de personas jóvenes y adultas, fuerza de trabajo, características socioeconómicas, diferenciación social.

\begin{abstract}
The objective of this work is to analyze to what extent the growing differentiation of the workforce affects the student body of secondary education for young and adults persons in Argentina in recent years. For this, different surveys performed by INDEC are used to carry out a statistical approach about the socioeconomic characteristics of EDJA secondary school student body throughout the country, seeking to determine the degree to which their reproduction conditions are mediated by situations such as unemployment, precarious employment and poverty, among other aspects. The results show that these students generally belong to sectors that are highly affected by the growing differentiation of the labor force, which is manifested in that they have more deteriorated living conditions than the average adult population.
\end{abstract}

Key words: secondary education; education for young and adults persons; workforce; socioeconomic characteristics; social differentiation

\section{Resumo}

O objetivo deste trabalho é analisar em que medida a crescente diferenciação da força de trabalho afeta a ele corpo estudantil da educação secundaria para pessoas jovens e adultas, na Argentina, nos últimos anos. Para isso, diferentes pesquisas realizadas pelo INDEC são utilizadas para realizar uma abordagem estatística sobre as características socioeconômicas do corpo discente do secundário da EDJA em todo o país, buscando determinar o grau em que suas condições de reprodução são mediadas por situações como desemprego, precariedade do emprego e pobreza, entre outros aspectos. Os resultados mostram que esses alunos e alunas geralmente pertencem a setores altamente afetados pela crescente diferenciação da força de trabalho, o que se manifesta em que tem condições de vida mais degradadas do que a média da população adulta.

Palavras-chave: educação secundaria; educação de pessoas jovens e adultas; força de trabalho; características socioeconômicas; diferenciação social. 


\section{Introducción}

De la mano de profundas transformaciones económicas a nivel mundial, en Argentina, se han producido, durante las últimas décadas, importantes cambios en las condiciones de vida de trabajadores y trabajadoras, que condujeron a una mayor diferenciación de la fuerza de trabajo. De esta manera, mientras que una porción de la fuerza laboral continuó reproduciéndose en circunstancias normales, otra lo hizo de modo cada vez más degradado, viéndose afectada por el incremento de la pobreza, la desocupación y la precariedad laboral, entre otras cuestiones (Iñigo Carrera, 2005; Kornblihtt et al., 2014; Seiffer y Rivas, 2017).

En lo que se refiere puntualmente a las particularidades socioeconómicas del estudiantado del secundario dentro de la educación de jóvenes y adultos (EDJA) en Argentina, existen autores/as que analizan este tema desde la demanda potencial de quienes están en situación de acceder a esta modalidad (Riquelme et al., 2018; Sirvent, 2005), como también otros/as que llevan adelante trabajos de campo circunscriptos a ciertos ámbitos geográficos donde se desarrollan sus procesos educativos (De la Fare, 2013; González, 2017; Montesinos et al., 2010). Sin embargo, aunque estas investigaciones realizan valiosas contribuciones, presentan ciertos límites para dar cuenta, en el plano cuantitativo, de las condiciones de reproducción del alumnado que, en efecto, concurre a la secundaria de la EDJA en todo el territorio nacional.

En línea con lo dicho, este trabajo tiene como objetivo analizar en qué medida esta creciente diferenciación de la fuerza de trabajo afecta al alumnado de la educación secundaria de personas jóvenes y adultas en Argentina en los años recientes. Para ello, se realiza una aproximación estadística acerca de las características socioeconómicas de los y las estudiantes en todo el país, buscando determinar cuál es el grado en que sus condiciones de reproducción están degradadas. Para comenzar, se examina, de modo general, la situación de la fuerza de trabajo en Argentina. A continuación, se abordan estudios previos de otros autores que toman en cuenta las particularidades socioeconómicas del alumnado del secundario de la EDJA en el país. Finalmente, se lleva a cabo un acercamiento cuantitativo con base en los relevamientos de la Encuesta Anual de Hogares Urbanos (2014), la Encuesta Permanente de Hogares (2003-2018) y el Censo Nacional de Población, Hogares y Viviendas (2010), realizados por el Instituto Nacional de Estadística y Censos (INDEC).

\section{Transformaciones en las condiciones de reproducción de la fuerza de trabajo en Argentina durante las últimas décadas}

Una variedad de estudios señala que la creciente diferenciación de la fuerza de trabajo ocurrida a lo largo de las últimas décadas dio como resultado que una parte de los asalariados pasaran a formar parte de la población relativamente sobrante para las necesidades inmediatas de valorización del capital y a tener condiciones de vida cada vez más deterioradas (Iñigo Carrera, 2005, Graña, 2012, Seiffer y Rivas, 2017). 
Esto se expresó, por ejemplo, en que el salario promedio tendió a descender desde su pico máximo en 1974, en especial durante las profundas crisis que atravesaron a la economía argentina (Iñigo Carrera, 2007; Kennedy, 2014; Kornblihtt et al., 2014). Por otro lado, la desocupación se elevó hasta su momento más álgido por los efectos de la crisis del 2001 y, aunque luego, en el siglo XXI, el empleo creció, los niveles de desempleo se mantuvieron en niveles altos (Arceo et al., 2008; Donaire, 2019; Poy y Salvia, 2017). A esto le acompañó un alza de la subocupación y el cuentapropismo, que se volvieron fenómenos regulares durante las últimas décadas (Burachik, 2012; Donaire, 2007; Villarreal, 1985).

Del mismo modo, y con particular fuerza durante la década de 1990, se amplió la precarización laboral con reformas legislativas que sancionaron el período de prueba, la contratación a tiempo determinado, la disminución de las indemnizaciones y la reducción de los aportes patronales (Lavopa, 2005; Lindenboim, 2001; Marticorena, 2005). Esta precarización fue aplicada, sobre todo, por las empresas de menor tamaño, que precisan recurrir a un mayor ataque a los costos laborales para subsistir (Espro y Zorattini, 2012). Entre otros aspectos, esto causó una ampliación de las diferencias entre trabajadores/as registrados/as y no registrados/as: mientras que los/as primeros/as lograron conservar un cierto poder adquisitivo a lo largo de las últimas décadas, los/as segundos/as sufrieron un retroceso constante de sus ingresos (Beccaria y Groissman, 2015; Kornblihtt et al., 2014; Salvia, 2010).

Todo ello condujo también a un incremento de la pobreza, como expresión de que una parte más grande de la población no llega a alcanzar los ingresos mínimos necesarios (Arakaki, 2015a; Beccaria, 2007; Kennedy y Graña, 2010). De hecho, en tal medida, se dio el empeoramiento de las condiciones de vida de una parte de la fuerza de trabajo, por lo que muchos hogares con ocupados se encuentran por debajo de la línea de pobreza. Tener un empleo dejó de ser garantía de estar por fuera de la pobreza (Cazón et al., 2016).

En cuanto al gasto público en servicios sociales, en sintonía con estos cambios, cobró más peso la asistencia social, a la vez que las políticas sociales pasaron a focalizarse cada vez más en la población empobrecida, para la cual aparece, en muchos casos, como una compensación salarial, sea para paliar circunstancias de desocupación o la de aquellos sectores cuyos ingresos fueran insuficientes para costear las necesidades mínimas de reproducción. De este modo, no solo dentro del gasto social total se dio un incremento relativo de la asistencia, sino que además de las políticas sociales en general tendieron a asistencializarse y quedar centradas en los sectores más afectados por la pobreza (Falappa y Andrenacci, 2009; Seiffer, 2013). Así, por ejemplo, se implementaron, de manera creciente, una diversidad de planes sociales de transferencia condicionada de ingresos, que posibilitaron la supervivencia de las fracciones más pauperizadas de la clase trabajadora (Cruces y Gasparini, 2009; Grassi y Alayón, 2005; Seiffer y Rivas, 2017).

La diferenciación de la fuerza de trabajo, de acuerdo con las demandas de la acumulación de capital, se manifestó también en el acceso a la educación (Hirsch, 2020; Iñigo y Rio, 2017; Mendonça y Pérez Trento, 2020). No casualmente, durante las últimas décadas, el sistema educativo argentino ha estado atravesado por fuertes procesos de diferenciación interna, a partir 
de la descentralización de la educación hacia las jurisdicciones, la desregulación del sector privado y la consolidación de circuitos escolares con ofertas y calidades muy distintas en todo el territorio nacional (Acosta, 2012; Braslavsky, 1989; Tiramonti, 2009). De esta manera, los sectores más empobrecidos son los que, en general, asisten a los circuitos educativos de menor calidad, a la vez que exhiben peores desempeños escolares y presentan mayores dificultades para completar la educación obligatoria (Ibánez Martín, 2017; Riquelme et al., 2018; Tiramonti, 2019).

\section{Características socioeconómicas del estudiantado de la educación secundaria para personas jóvenes y adultas}

Si se toma esta cuestión en un sentido amplio, para algunos/as autores/as, el origen socioeconómico del estudiantado es lo que determina en efecto la especificidad de la educación de personas jóvenes y adultas, como afirman, por ejemplo, Brusilovsky y Cabrera (2006). Para estas autoras, la EDJA se centra en la formación de "sectores populares" que han quedado por fuera del sistema escolar. Es entonces que, a diferencia de quienes delimitan a sus alumnas/os desde lo etario, Brusilovsky (1995) señala que, en Argentina y América Latina, la misma se ha referido a la educación de personas jóvenes y adultas definidas como carenciadas, obreras/os, pobres, trabajadoras/es, etc. En el mismo sentido, Rodríguez (2009), por su parte, afirma también que el término adulto oculta el verdadero sujeto que accede a la EDJA: un "marginado pedagógico", perteneciente a los "sectores subordinados", indicando que la especificidad del estudiantado que asisten a la modalidad no se debe a su edad, sino a sus condiciones socioeconómicas. Es así que tanto la pobreza como la situación laboral son elementos centrales en la secundaria para este grupo (Rodríguez, 2008).

Un modo de aproximarse a las condiciones de vida de este grupo de la secundaria de la EDJA en Argentina puede hacerse de forma indirecta, a través de indicadores que muestren las particularidades de quienes potencialmente estarían en circunstancias de acceder a la modalidad. Es decir, de aquellas personas jóvenes y adultas que no han llegado a finalizar el nivel secundario en las distintas partes del país. En este caso, autoras como Sirvent (2005) han avanzado en el reconocimiento del vínculo entre la pobreza y los bajos niveles escolares. Esta "pobreza educativa" afecta cada vez a más individuos, ya que se agudiza con el empobrecimiento generalizado de la sociedad argentina.

Similares observaciones han hecho Riquelme et al. (2018) acerca de la población potencial que accede a la EDJA. Estas investigadoras explican que, si bien se ha dado una expansión en el acceso al secundario en jóvenes y adultos/as, aún existen importantes divergencias entre hogares si se tienen presentes indicadores como las necesidades básicas insatisfechas (NBI) o los ingresos per cápita.' Según estas autoras, quienes viven en hogares con NBI demuestran mayores niveles de deserción escolar antes de haber finalizado el nivel secundario que quienes no tienen necesidades básicas insatisfechas. Estas disparidades se hacen más notables si se comparan los 
ingresos del hogar, lo cual muestra que los más pobres son los que menos logran completar la educación sancionada como obligatoria por el Estado.

Otros autores rescatan el concepto de "nivel educativo de riesgo" (NER) para referirse a las personas de 15 años o más que dejaron de asistir a un establecimiento escolar llegando a alcanzar la primaria incompleta, primaria completa o secundaria incompleta (Topasso et al., 2015). Estos sujetos en "situación educativa de riesgo" son considerados como destinatarios potenciales de la EDJA. A partir de una lectura sobre el NER en todo el país, estas investigaciones señalan notorias diferencias a nivel nacional, mostrando, a la vez, que son los hogares pobres los más afectados por la deserción escolar prematura.

Una manera distinta de aproximarse a este objeto de estudio es aquella que surge de las producciones que han llevado adelante trabajos de campo para observar los rasgos distintivos de la población que asiste a la modalidad. En una investigación dedicada específicamente al alumnado del secundario de la EDJA, De la Fare (2013) muestra, por ejemplo, la diversidad de situaciones laborales de los/as destinatarios/as. Unos/as están ocupados/as en empleos informales, mientras que otros/as se encuentran desocupados/as o subocupados/as. Algunas de estas personas se desempeñan como asalariadas en empresas privadas de la industria o el comercio, así como en el ámbito estatal, mientras que otras efectúan actividades de servicios tales como el cuidado o la limpieza doméstica, reparación de electrodomésticos, etc., o tienen pequeños emprendimientos, como puede ser un comercio. Este tipo de inserción laboral ha sido reconocida por otros autores como Montesinos et al. (2010).

Resultados similares son señalados por distintos análisis sobre el Plan de Finalización de Estudios Primarios y Secundarios para Jóvenes y Adultos II, más conocido como FinEs II. ${ }^{2}$ En este caso, en los trabajos de campo en ámbitos locales, también se reconoce que quienes acceden a la EDJA están atravesados/as por la precariedad laboral, los bajos salarios, el cuentapropismo y la desocupación, entre otros aspectos (González, 2017; Pecarrere, 2014; Sucunza, 2016). Nuevamente, el servicio doméstico, las changas, el trabajo independiente o en ramas como la construcción, el comercio o la industria manufacturera se hacen presentes en los estudios de campo de estos autores.

En síntesis, las investigaciones mencionadas hacen aportes valiosos para poder comprender las características socioeconómicas del alumnado de la EDJA. En el caso de aquellas que examinan la demanda potencial, se destaca el hecho de que quienes se encuentran en condiciones de acceder a la modalidad viven en hogares que están, por lo general, debajo de la línea de pobreza o que presentan necesidades básicas insatisfechas. No obstante, el límite que se encuentra en este tipo de aproximaciones es que brindan una lectura global que abarca a todos/as los/as que, potencialmente, podrían estudiar en la EDJA, pero no a quienes de manera efectiva lo hacen. Por lo tanto, se hace necesario avanzar en análisis más concretos sobre quienes asisten a la educación de personas jóvenes y adultas. En contraposición, las producciones referidas a estudios de caso posibilitan ver otros rasgos más específicos. Es decir, observan que son sujetos que, por ejemplo, se encuentran desocupados, o trabajando de manera precaria por 
bajos salarios en ciertas ramas de la economía. Sin embargo, al tratarse de investigaciones que tienen un carácter local, no queda definido en qué medida sus conclusiones son representativas o no del conjunto del alumnado de la EDJA a nivel nacional.

\section{Una aproximación estadística a las condiciones de vida de los alumnos de la educación secundaria de la EDJA en Argentina}

De acuerdo con el objetivo central planteado al comienzo de este trabajo, en este apartado, se busca mirar las particularidades socioeconómicas de los individuos que cursan sus estudios en la educación secundaria de jóvenes y adultos/as en Argentina en los años recientes a partir de relevamientos estadísticos nacionales. Se toma como muestra principal la Encuesta Anual de Hogares Urbanos (EAHU) Ilevada a cabo por el INDEC en el año 2014. Se elige esta fuente debido a que brinda información de manera sistemática y estandarizada acerca del mercado de trabajo urbano en el conjunto del territorio nacional, lo cual no es menor teniendo en cuenta que Argentina es un país donde la población urbana es alrededor del 91\% del total (Donaire et al., 2017). Para realizar una lectura sobre la evolución de la pobreza por ingresos, se escoge, por única vez, la Encuesta Permanente de Hogares (EPH) entre los años 2003 y 2018, ya que la EAHU solo se encuentra disponible entre los años 2010 y 2014. En el caso de la EPH, las características son similares a las de la EAHU, pero abarca únicamente 31 aglomerados urbanos (capitales de las provincias y localidades de más de 100.000 habitantes), llegando a cubrir el 70\% de la población total del país. Finalmente, para hacer mención de la pertenencia a hogares con Necesidades Básicas Insatisfechas, se toma en cuenta el Censo Nacional de Población, Hogares y Viviendas del año 2010, que abarca a la totalidad de la población argentina de ese año, debido a que es la fuente con más alcance para tomar en cuenta las NBI.

Para acotar el universo de los estudiantes de la EDJA a analizar, se tomó como referencia a toda la población de entre 20 y 64 años inclusive, cuyo máximo nivel educativo se corresponda con la primaria completa o la secundaria incompleta, y que se encuentre concurriendo a un establecimiento escolar. La edad mínima fue elegida para no incorporar a alumnos de entre 12 y 19 años que pudieran estar participando de la secundaria común. La edad máxima se seleccionó asumiendo los 65 años como la edad promedio de jubilación, en la cual las personas se retiran, por lo general, del mercado laboral. Como grupo de control, se escogió a la población adulta total de la misma edad en todo el país, para poder observar si las características del alumnado del secundario de la EDJA son específicas de dicho grupo o no, y en qué medida son iguales o difieren de las del conjunto de la población adulta. Haciendo este recorte con la EAHU del 2014, la muestra dio un total de 403.345 asistentes al nivel medio de la EDJA, cifra no menor si se tiene en consideración que el número total de alumnos/as de la secundaria de jóvenes adultos/as relevados por la Dirección Nacional de Información y Evaluación de la Calidad Educativa (DINIECE) para ese año era de 539.490 . 
Resulta relevante hacer algunas apreciaciones metodológicas acerca de las divergencias en las cifras. En primer lugar, una fracción de esa diferencia se debe al hecho de que los relevamientos de la DINIECE, que se realizan a partir de la información que brindan las instituciones escolares, toman como parte de la EDJA también a individuos que concurren a la modalidad entre las edades de 12 y 15 años (6.079 alumnos en 2014) y de 16 a 19 años (199.243 alumnos en 2014), y que quedan excluidos del presente estudio por las razones antes mencionadas. Por otro lado, el relevamiento de la DINIECE del 2014 no incorpora la matrícula de programas de terminalidad educativa con fuerte peso en la población como es, por ejemplo, el caso del plan FinEs, que cuenta con miles de estudiantes en todo el territorio nacional.

A la vez, las muestras de la EAHU y la DINIECE son llevadas a cabo en dos momentos distintos del año. Mientras que la primera se hace a lo largo de las doce semanas del tercer trimestre del año, la segunda se concreta en abril y toma las edades de los alumnos al mes de junio (o excepcionalmente a diciembre del año pasado, dependiendo del período de funcionamiento del establecimiento). Esto puede causar, en primer lugar, que alumnos que figuran en el relevamiento educativo en abril hayan desertado de la EDJA cuando se realiza el relevamiento del INDEC entre julio y septiembre. En segundo lugar, que algunas/os estudiantes que figuran en la muestra de la DINIECE con 19 años al mes de junio, tengan 20 años durante el tercer trimestre del mismo año. Por último, los relevamientos educativos de la DINIECE alcanzan a todo el territorio de Argentina, mientras que la EAHU solo abarca las zonas urbanas, con lo cual existe la posibilidad de que una parte de la matrícula no sea captada por esta última (aunque, por lo general, la EDJA en Argentina se desarrolla en espacios urbanos). Más allá de estas diferencias, los relevamientos efectuados por el INDEC brindan información significativa para poder comprender las condiciones de vida de una porción representativa de los sujetos que acceden al secundario dentro de la modalidad de jóvenes y adultos/as en la totalidad de Argentina.

Para comenzar, si se analizan los datos que la EAHU presenta en el año 2014, puede verse que un poco menos de la mitad de los y las asistentes al nivel medio de la EDJA se encuentran ocupados/as, mientras que la mitad se hallan inactivos/as y menos del 10\% están desocupados/as. ${ }^{3}$ El gran peso de los/as inactivos/as, superior al del conjunto de la población adulta, probablemente se debe a que la mayor parte figura en el relevamiento como estudiantes, y que tampoco tienen un empleo. 


\section{Gráfico 1}

Condición de actividad en porcentajes. Alumnos del secundario de la EDJA y población adulta total. Argentina, 2014

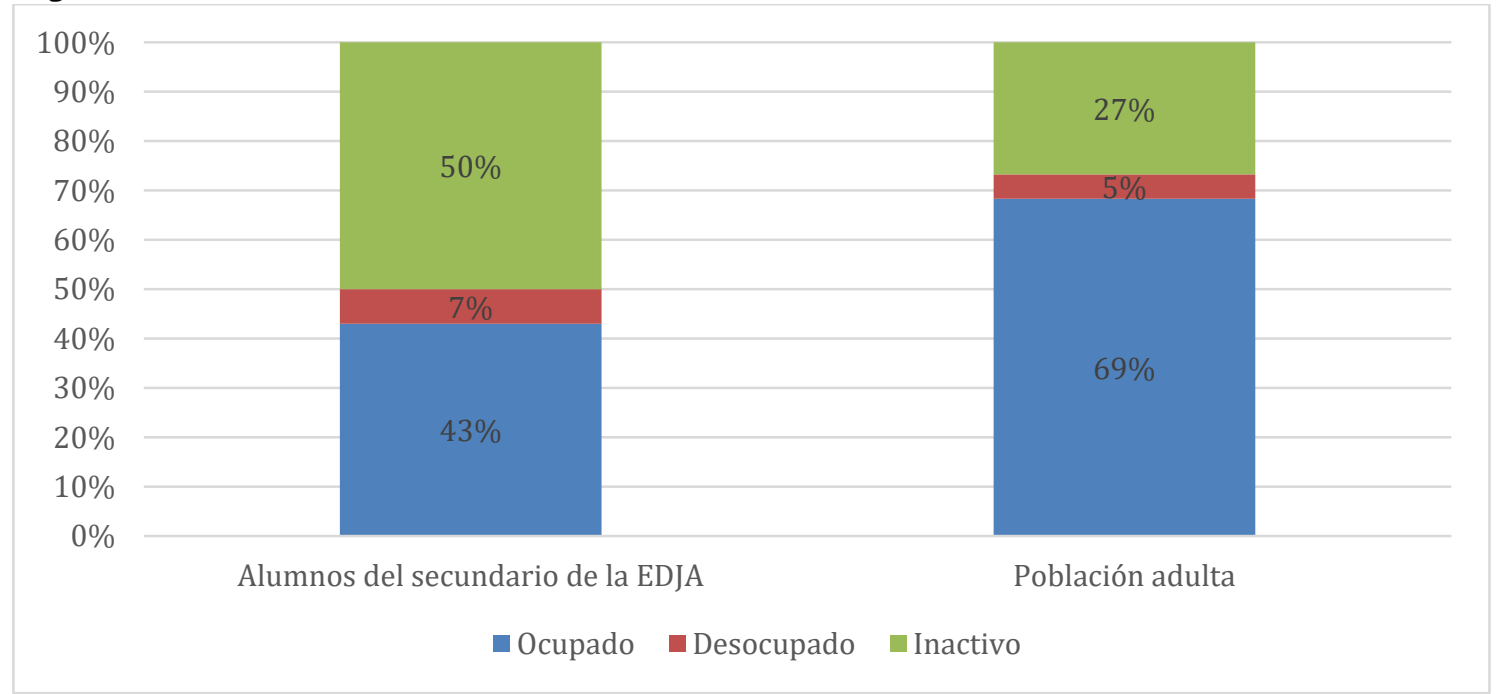

Fuente: elaboración propia con base en EAHU-INDEC.

Continuando con la exploración, si se centra la mirada en los y las asistentes al nivel medio de la EDJA que están ocupadas/os, la enorme mayoría de ellas/os (un 79\%) figuran como asalariadas/os, mientras que casi un quinto (18\%) como cuentapropistas. Patrones/as y trabajadores/as familiares sin remuneración son marginales en esta muestra. Esto no difiere en gran medida de los promedios generales de la población adulta. Por otro lado, entre las ramas en las que se ubican los y las asistentes a la EDJA ocupados/as, las que se destacan son, por orden de relevancia, la de comercio (21\%), servicio doméstico (17\%), industria manufacturera (11\%), construcción (10\%) y alojamiento y servicio de comidas (8\%). Entre ellas, abarcan casi al $70 \%$ de las/os ocupadas/os dentro del alumnado del nivel medio para personas jóvenes y adultas. Esto no resulta una gran sorpresa, ya que son, en gran medida, algunas de las ramas de la economía que más empleo absorbieron a lo largo del siglo XXI en Argentina (Beccaria y Maurizio, 2017; Coatz y Schteingart, 2016). Aquí, por ende, tampoco parece haber grandes diferencias si se cotejan estos datos con el conjunto de la población adulta, a excepción por el caso del servicio doméstico, que alcanza ocho puntos más para los y las estudiantes del secundario para personas jóvenes y adultas.

En lo que respecta a la intensidad de la ocupación principal de los y las asistentes al secundario de la EDJA, un poco más de la mitad (54\%) declaran tener ocupación plena. Por otro lado, aproximadamente un cuarto del estudiantado (26\%) se encuentra sobreocupado y, casi un quinto (19\%), subocupado (del cual un $14 \%$ desea trabajar más horas). Aquí, no parece haber una diferencia muy significativa entre este grupo y el conjunto de las/os adultas/os. En resumen, lo que puede verse es que, dentro del conjunto del alumnado estudiado, la situación laboral es 
bastante heterogénea. Así, mientras que una parte logra obtener un empleo con la cantidad de horas consideradas normales, otra debe trabajar largas jornadas para poder subsistir o, por el contrario, no es absorbida plenamente por el mercado de trabajo y, por ende, no logra conseguir un empleo con la cantidad de horas deseada.

En cuanto a las personas desocupadas, la mayor parte (91\%) declaran haber trabajado antes y gran parte de ellas (74\%) afirman que buscaron trabajo de modo activo. Esto es similar para ambos grupos analizados, con algunos puntos más para el caso de asistentes de la EDJA. Por otro lado, casi la mitad de las personas desocupadas (47\%) afirma haber realizado alguna changa o trabajo temporal en el tiempo en el que buscaron empleo. Tampoco en este caso se observan diferencias notorias. Lo que puede decirse al respecto es que, en este caso, se trata de una porción de la fuerza laboral que es expulsada del mercado de trabajo, y que debe recurrir a trabajos temporales, normalmente con bajas remuneraciones, para poder obtener ingresos.

En cuanto al tamaño de los establecimientos donde se desempeñan los ocupados relevados, más de la mitad de quienes concurren a la educación secundaria de jóvenes y adultos lo hacen en pequeñas empresas de hasta 5 empleados. Solo un 19\% están ocupados en empresas de más de 40 empleados. Se trata entonces de personas que trabajan sobre todo en pequeñas y medianas empresas, siendo este aspecto 11 puntos más alto que el de la población adulta total. Por la especificidad de la acumulación de capital en Argentina, los pequeños y medianos capitales han tenido un gran peso en el desarrollo de la economía local (Espro y Zorattini, 2012; Graña, 2012; Iñigo Carrera, 2005). Esto resulta relevante ya que, como se explicó con anterioridad, son este tipo de capitales los que mayormente explotan a sus empleados por bajos salarios y en condiciones precarias.

\section{Gráfico 2}

Tamaño de la empresa en que trabaja en porcentajes. Alumnado del secundario de la EDJA y población adulta total. Argentina, 2014

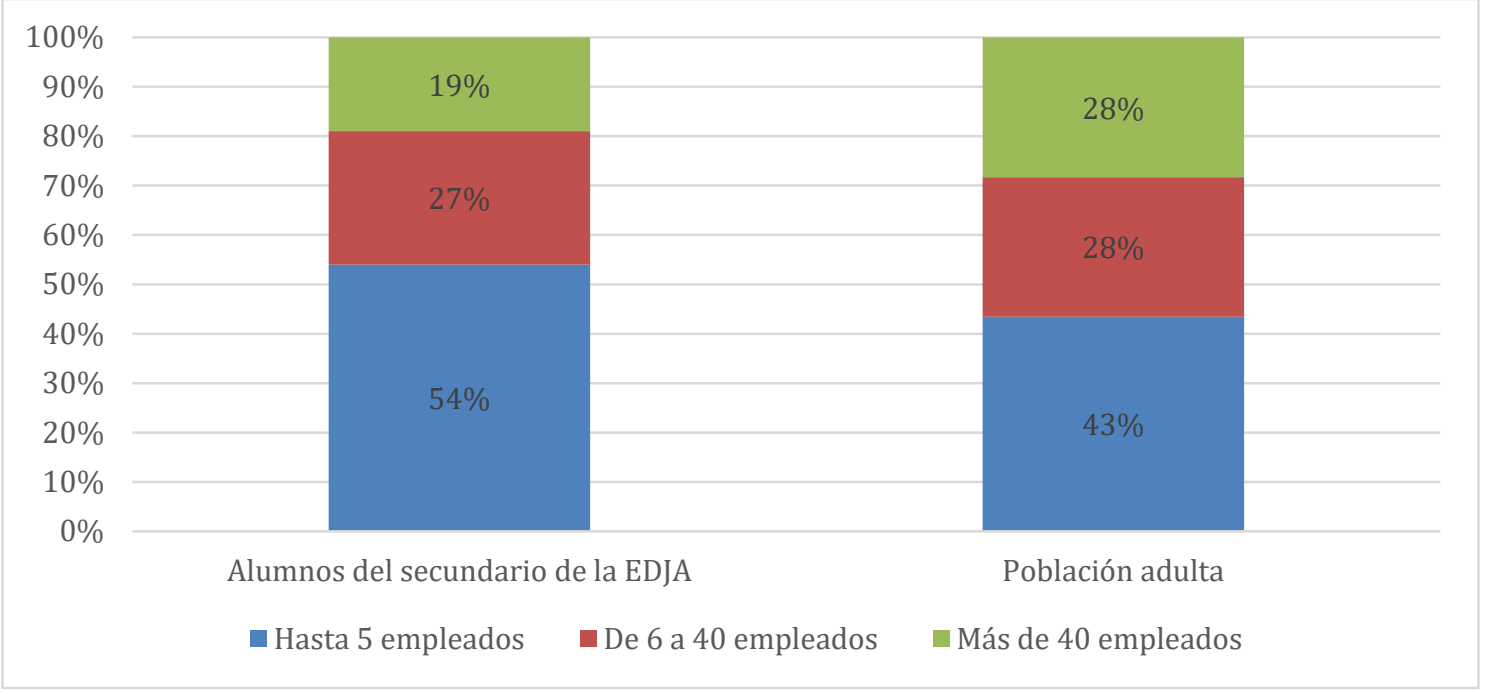

Fuente: elaboración propia con base en EAHU-INDEC. 
En relación con lo dicho antes, existen varios indicadores que señalan que quienes asisten a la secundaria para personas jóvenes y adultas tienen trabajos que, en general, son más precarios que los del conjunto de la población adulta. Usualmente, para distinguir entre trabajadores/as protegidos/as y precarios/as, se utiliza el acceso o no a descuento jubilatorio (Arakaki, 2015b). Al respecto, solo dos de cada cinco individuos empleados que realizan sus estudios secundarios en la EDJA tienen empleos con dicho descuento. La diferencia aquí, en relación con la población adulta, es de 25 puntos. A la misma conclusión se llega si se observa el acceso a un trabajo que provea obra social, licencia con días pagos por enfermedad, derecho a vacaciones pagas o sueldo anual complementario (más conocido como aguinaldo). Resulta revelador también que un $21 \%$ de quienes concurren al nivel medio para personas jóvenes y adultas tienen un empleo temporal. Este número es un tanto más alto que el de la población adulta total, que se ubica en un $13 \%$ en el año 2014. De esta manera, puede verse que se trata, en gran medida, de trabajadores que venden su fuerza de trabajo de manera precaria, abaratando los costos de las empresas que los contratan, obteniendo así una mayor ganancia. No está de más repetir que esta cuestión no puede desligarse del tamaño de las empresas que, en general, contratan a estos individuos.

\section{Gráfico 3}

Asalariado con descuento jubilatorio en porcentajes. Alumnado del secundario de la EDJA y población adulta total. Argentina, 2014

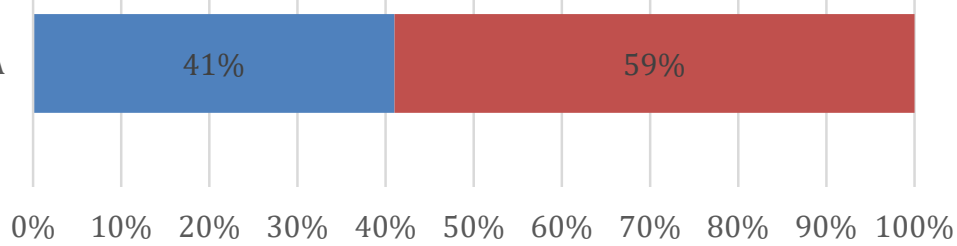

—Sí — No

Fuente: elaboración propia con base en EAHU-INDEC.

En línea con lo dicho antes, si se compara el salario de la ocupación principal de los individuos relevados, también pueden verse claras asimetrías. Así, para el año 2014, tres cuartos de las personas asistentes al secundario de la EDJA ganaban menos de un salario mínimo y solo el 1\% ganaba dos salarios mínimos o más. En el caso de la población adulta total, la cantidad de individuos que ganaban menos de un salario mínimo era del $45 \%$ y un $18 \%$ ganaban más de dos salarios mínimos o más. ${ }^{4}$ Esto resulta llamativo ya que, como se dijo antes, el $80 \%$ estaban 
ocupados de manera plena o sobreocupados. Es decir, no se trata de que ganen poco porque trabajen poco, sino más bien de que ganan poco a pesar de trabajar mucho. De nuevo, se debe recordar que los ingresos de los asalariados deben ser analizados a la luz de los capitales que los explotan que, como se vio antes, en su gran mayoría, son pequeñas y medianas empresas.

\section{Gráfico 4}

Salario de la ocupación principal. Alumnado del secundario de la EDJA y población adulta total. Argentina, 2014

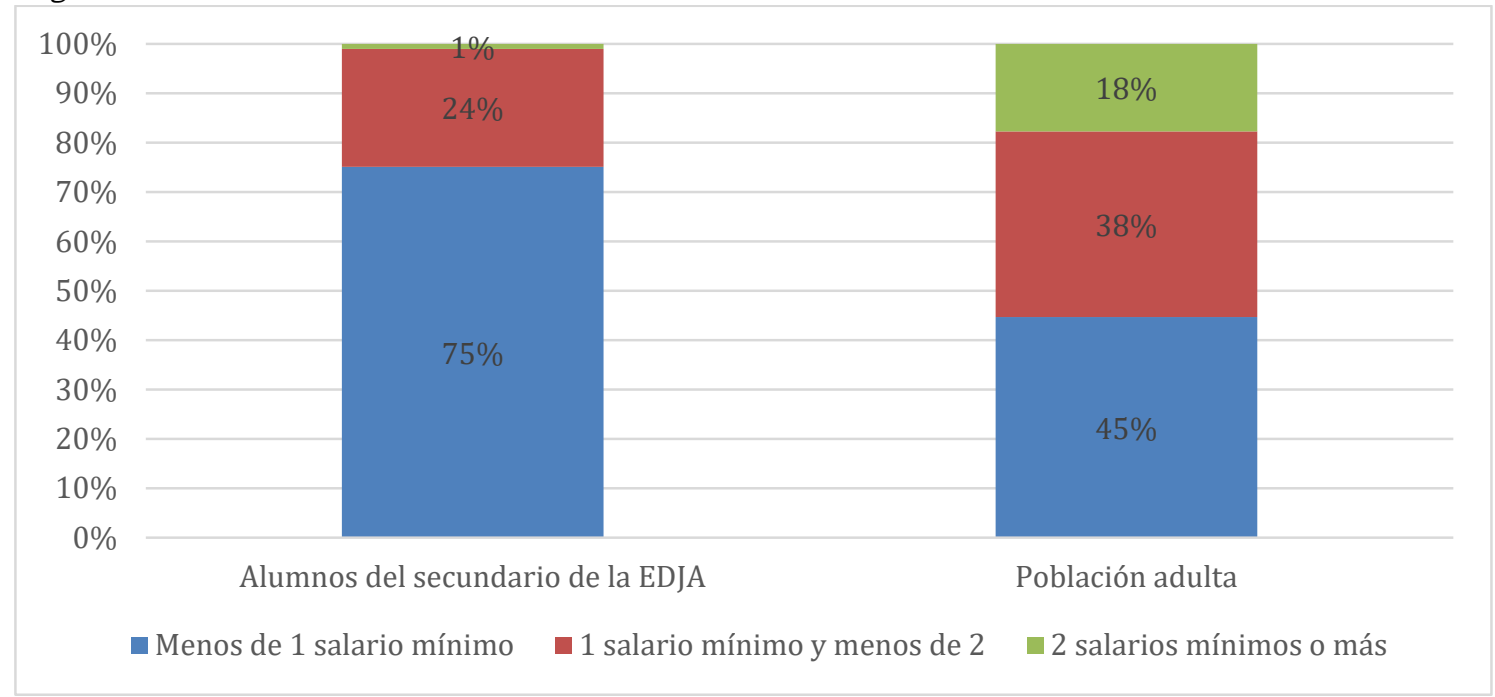

Fuente: elaboración propia con base en EAHU-INDEC.

En consonancia, si se observa la evolución de la pobreza por ingresos desde el 2003 hasta el año 2018, puede verse que las personas asistentes al secundario de la EDJA pertenecen a hogares que, en general, son más pobres que los del conjunto de la población adulta. De ese modo, al comienzo de la recuperación económica del 2003, más del 70\% de quienes concurrían al secundario para personas jóvenes y adultas eran pobres y, aún en el mejor de los años, ese número no baja del 33\%. Hacia el final de la serie, en un nuevo contexto de crisis de la economía argentina, ese número vuelve a dispararse a más del 50\% para quienes realizan la secundaria en la EDJA.

Según el INDEC, la pobreza existe cuando un hogar no puede alcanzar ingresos equivalentes al valor de la Canasta Básica Total (que incluye alimentos, así como también otros servicios no alimentarios tales como la educación, transporte o vestimenta). Esto es una clara manifestación de que quienes concurren al secundario para personas jóvenes y adultas viven, en general, en condiciones que no les permiten reproducirse con normalidad, no logrando alcanzar un consumo de bienes mínimo. Desde ya, esta cuestión no puede desligarse en absoluto del empobrecimiento que ha atravesado a la población en Argentina durante las últimas décadas, como se explicó antes. Al transformar a una parte de la fuerza de trabajo en sobrante para sus necesidades inmediatas, el capital la priva así de su capacidad para poder participar del consumo 
social pleno, aun cuando de hecho tenga un empleo. Esto genera que, a lo largo del tiempo, la población empobrecida vea su existencia deteriorada. En relación con esto, tal como muestra el Censo Nacional de Población, Hogares y Viviendas del 2010, ese año, el 11,01\% de quienes asistían al secundario de la EDJA vivían en hogares con al menos un indicador de NBI, número muy similar al del conjunto de la población adulta.

\section{Gráfico 5}

Evolución de la pobreza por ingresos en porcentajes. Alumnado del secundario de la EDJA y población adulta total. Argentina, 2003-2018

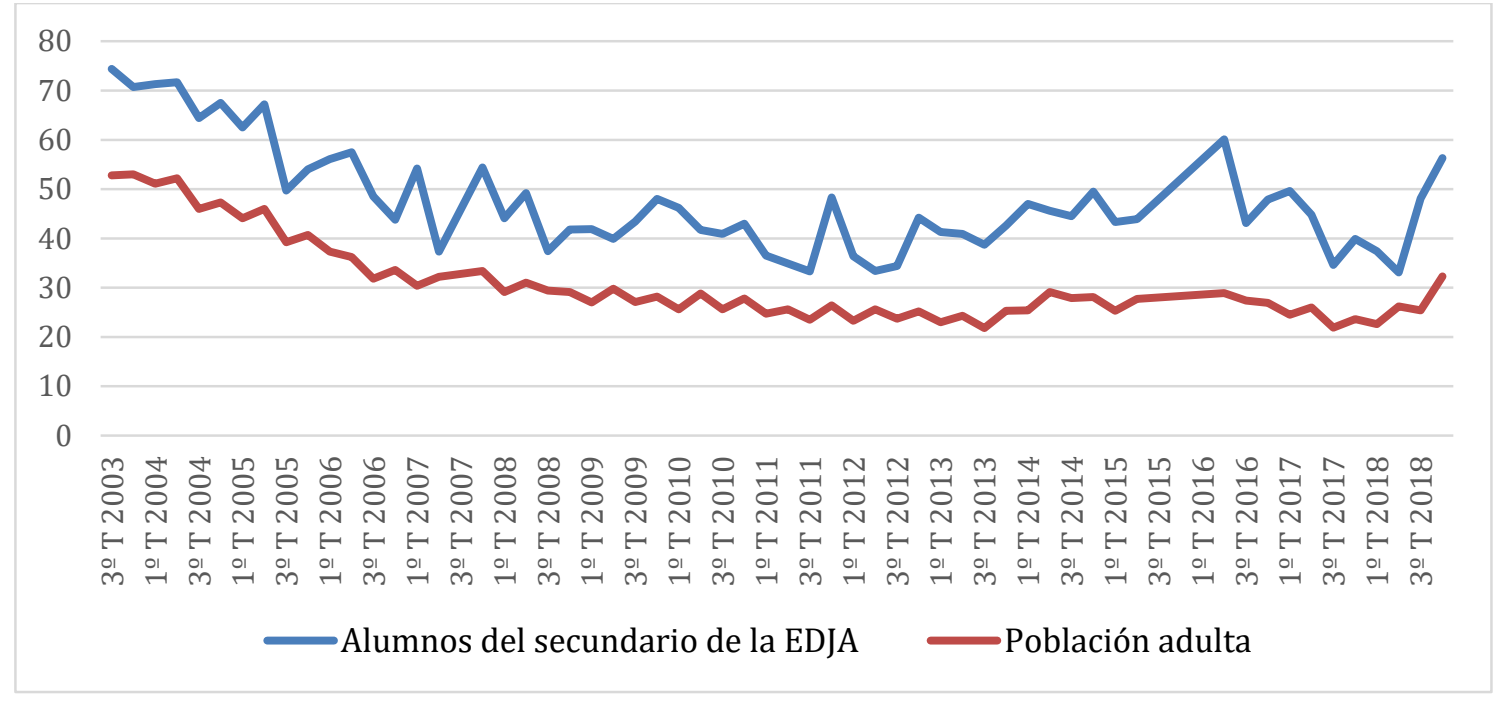

Fuente: elaboración propia con base en EPH-INDEC y Zack et al., 2017. Aclaración: se omitió la información del tercer trimestre del 2007, tercer y cuarto trimestre del 2015 y primer trimestre del 2016, ya que la misma no está disponible.

Como contracara, en lo que se refiere al acceso a la asistencia social por parte del Estado o instituciones como la iglesia, también aquí se trata de una cifra más elevada en el caso de las personas asistentes a la secundaria de la EDJA. De esa manera, en 2014, el 37\% de estas recurrían a subsidios o ayuda en forma de dinero o mercadería para su subsistencia, número que, en el total de las personas adultas, se ubicaba en un $19 \%$. De este modo, frente al empobrecimiento de la población en Argentina, la asistencia aparece cada vez con más fuerza como un salario indirecto para los hogares, sea porque sus miembros no tienen trabajo, o porque con los trabajos que tienen no llegan a alcanzar los ingresos necesarios para poder subsistir. Hasta tal punto esto es así que, incluso una gran parte de las instituciones educativas en el país, han pasado a cumplir cada vez con más fuerza funciones asistenciales, las cuales implican incluso brindar alimentos de manera directa a las familias de los alumnos. 


\section{Gráfico 6}

Acceso a subsidios o ayuda social en dinero o mercadería de instituciones públicas o privadas en porcentajes. Alumnado del secundario de la EDJA y población adulta total. Argentina, 2014

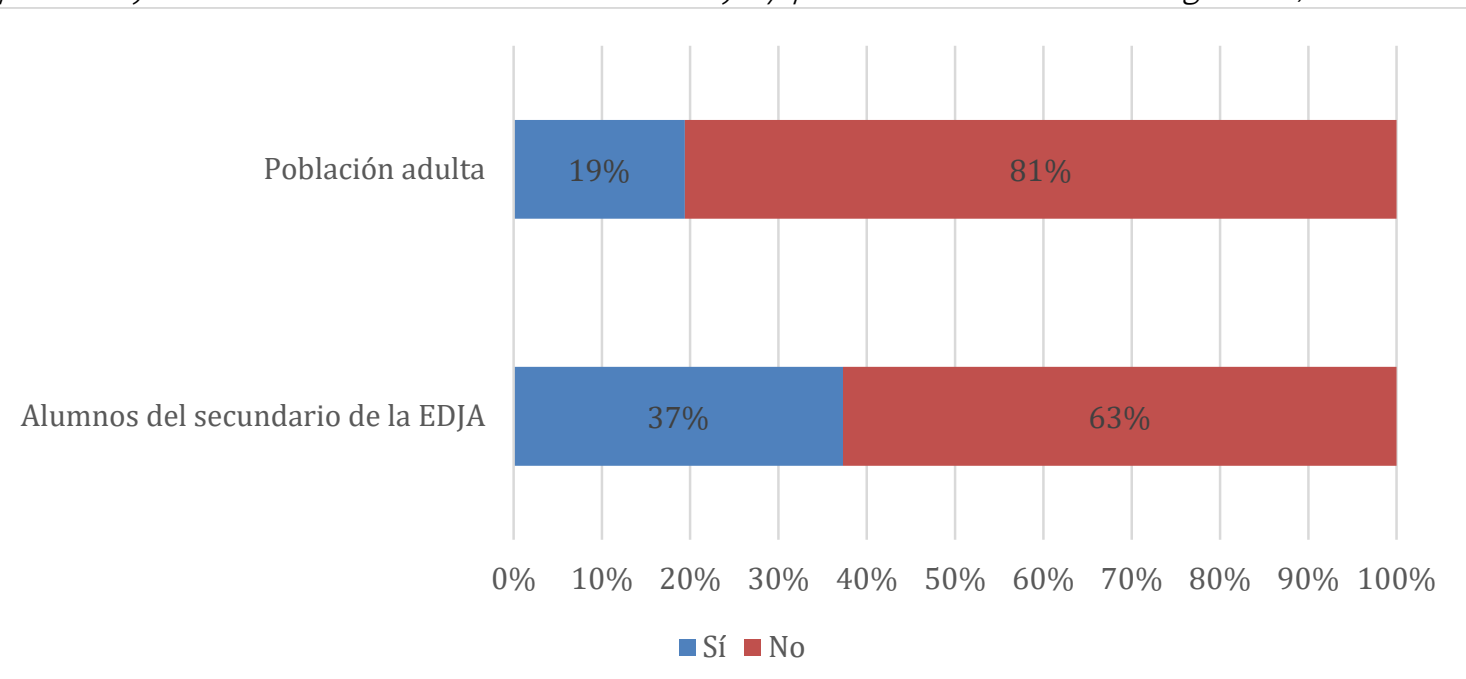

Fuente: elaboración propia con base en EAHU-INDEC.

Haciendo una síntesis de la información analizada sobre el estudiantado del secundario para personas jóvenes y adultas, puede verse que, casi la mitad de los individuos relevados, se encuentran ocupados, siendo la mayor parte de ellos asalariados. Las ramas donde se desempeñan son, sobre todo, algunas de las que más empleo absorbieron en los últimos años en Argentina (comercio, servicio doméstico, manufactura, construcción, etc.). La mayoría de ellos trabajan en pequeñas y medianas empresas. De las personas ocupadas, solo la mitad lo hace de manera plena, mientras que el resto se divide de modo relativamente equitativo entre subocupados y sobreocupados. A la vez, más de la mitad de las personas asalariadas están precarizadas, sin gozar de aguinaldo, aportes jubilatorios, obra social, vacaciones pagas, etc. Por otro lado, uno de cada cinco figuran realizando trabajos temporales. Al mismo tiempo, la mitad del alumnado relevado aparece en la muestra del INDEC como inactivo, ya que se dedican a estudiar y no buscan trabajo de modo activo. Dentro del minoritario grupo de los desocupados, la mayoría de ellos trabajaron con anterioridad y ahora se encuentran desempleados. De ellos, tres de cada cuatro dicen haber buscado empleo enviando su currículum o respondiendo/colocando avisos. Mientras tanto, casi la mitad hizo changas.

En cuanto al salario de la ocupación principal de quienes están empleados, tres de cada cuatro asistentes al secundario de la EDJA ganan menos del salario mínimo por su trabajo, a pesar de que, en gran medida, se desempeñan en jornadas normales o incluso más largas de lo común. En consonancia, se trata de una porción de la fuerza de trabajo que se reproduce con bajos ingresos. Es común que vivan en la pobreza, llegando esta a superar el $70 \%$ en el 2003 y no bajando del $33 \%$ en los mejores años. No es casual que un $37 \%$ de ellas/os recurran a la asistencia social en forma de ayudas en dinero o mercadería por parte de organismos públicos o privados. 
Estas características son coincidentes con lo que plantean los estudios previos sobre el estudiantado de la secundaria de personas jóvenes y adultas reseñados (tanto los que analizan la matrícula potencial, como los que realizan estudios de caso en espacios geográficos limitados), a los que el presente trabajo buscó aportar desde la estadística a nivel nacional.

\section{A modo de cierre}

Este escrito tuvo como objetivo analizar en qué medida la creciente diferenciación de la fuerza de trabajo afecta al alumnado de la educación secundaria de personas jóvenes y adultas en Argentina en años recientes. Para ello, se avanzó por distintos niveles de análisis. En primer lugar, se exploraron las condiciones del mercado de trabajo en el país durante las últimas décadas. Se reconoció así que, desde mediados de la década de 1970, se incrementó la fragmentación de la fuerza laboral. De este modo, mientras que una parte del colectivo de los trabajadores continuó reproduciéndose en condiciones normales, otra lo hizo de manera cada vez más degradada. En segundo lugar, se retomaron las investigaciones previas acerca de las condiciones de vida del alumnado del secundario de la EDJA, marcando sus contribuciones y posibles límites para dar cuenta de las características socioeconómicas de estos todo el territorio argentino. En tercer lugar, se llevó adelante una aproximación estadística utilizando distintos relevamientos desarrollados por el INDEC (en especial la EAHU del 2014, pero también la EPH entre 2003 y 2018 y el Censo Nacional de Población, Hogares y Viviendas del 2010), teniendo como meta determinar cuál es el grado en que sus condiciones de reproducción están mediadas por situaciones tales como la desocupación, la precariedad laboral y la pobreza, entre otros aspectos.

De acuerdo con lo observado, pudo verse que, tal como han explicado otras investigaciones, una gran parte de quienes asisten a la educación secundaria de personas jóvenes y adultas, en Argentina, viven en condiciones sumamente deterioradas, en promedio peores que los de la población adulta. Para el año 2014, esto se observó, por ejemplo, en que solo el 43\% tenían una ocupación, en que el $75 \%$ de quienes estaban ocupadas/os percibían bajos salarios en sus empleos, en que un $59 \%$ trabajaban en condiciones precarias, en que, ese año, más de un $40 \%$ estaba bajo la línea de pobreza o en que un $37 \%$ de ellos/as recurría a la asistencia social para poder subsistir. De lo visto a lo largo de este escrito, se desprende, también, que no puede entenderse la situación del estudiantado del nivel medio de la EDJA sin considerar el creciente empobrecimiento y diferenciación de la fuerza de trabajo que ha determinado el proceso de acumulación de capital durante las últimas décadas en el país. Es por ello que, en mayor o menor medida, estas características mencionadas son compartidas por una parte sustancial de la población adulta.

Diversas preguntas pueden derivarse de este análisis. Una de ellas, central para comprender el desarrollo de la EDJA, se origina en relación con los atributos con los que se forman los y las estudiantes que acceden a la modalidad. En este sentido, si la acumulación de capital en Argentina ha conducido a una mayor diferenciación de la fuerza de trabajo, ¿a qué necesidad responde la extensión general de su escolaridad? ¿La expansión del secundario para personas 
jóvenes y adultas en Argentina surge solo de las demandas de la población por obtener el título secundario para estar en mejores condiciones dentro del mercado laboral, o existen necesidades propias de la economía argentina de contar con ciertos conocimientos específicos en una fracción de la fuerza de trabajo que no estaría accediendo a ellos en la secundaria común? Este tipo de preguntas invitan a continuar explorando la evolución reciente de la educación para personas jóvenes y adultas en el país.

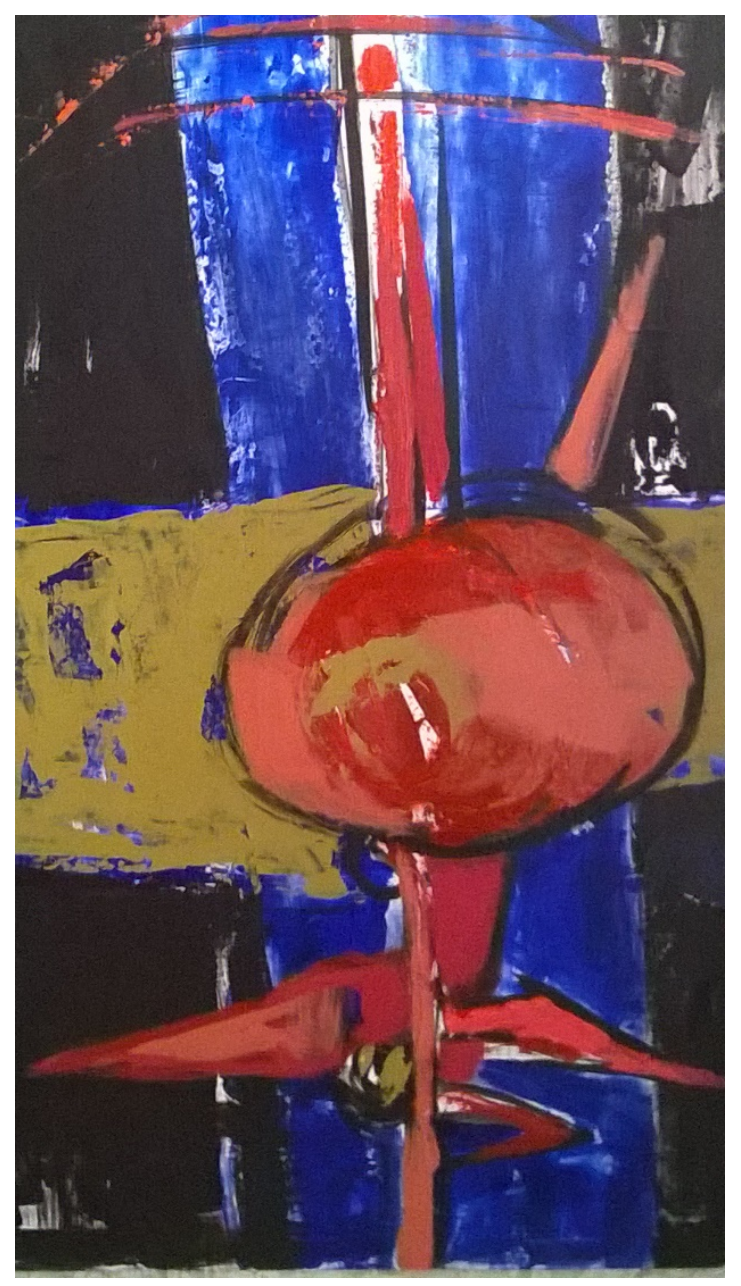

ST, acrílico. Noemí Fiscella

\section{Notas}

${ }^{1}$ Los indicadores de Necesidades Básicas Insatisfechas (NBI) se presentan en aquellos hogares que habitan en una vivienda de tipo inconveniente (pieza de inquilinato, vivienda precaria, etc.), que no cuentan con un cuarto de baño, que poseen al menos un niño en edad escolar que no asiste a la escuela o que tienen cuatro o más personas por miembro ocupado y en los cuales el jefe o jefa de hogar detenta un bajo nivel educativo. 2 El Plan de Finalización de Estudios Primarios y Secundarios II (FinEs II) es un programa educativo creado a nivel nacional para lograr que personas jóvenes y adultas que abandonaron sus estudios puedan completar la secundaria. Se presenta bajo la forma de un trayecto escolar presencial, con una menor carga horaria total 
que la escuela media común, contratos más precarios para el personal docente y la articulación con otras instituciones que funcionan como espacios escolares, tales como sindicatos, centros culturales, iglesias, etc. (Burgos, 2015; Viego, 2015).

${ }^{3}$ La fuente consultada considera como población económicamente activa a todas aquellas personas que tienen una ocupación (ocupados) o que no la tienen, pero están buscando tenerla de manera activa (desocupados). Por otro lado, define como inactivos a todos aquellos individuos que declaran no querer o no poder trabajar. En este último caso, se trata de un conjunto muy heterogéneo ya que la categoría inactivos puede abarcar tanto a jubilados como estudiantes, amas de casa, discapacitados, rentistas, etc. (Donaire et al., 2017).

${ }^{4}$ El salario mínimo sancionado por el Estado, en 2014, era de $\$ 4.400$.

\section{Bibliografía}

Acosta, F. (2012). La escuela secundaria argentina en perspectiva histórica y comparada: modelos institucionales y desgranamiento durante el siglo XX. Cadernos de Historia da Educação, 11(7), 131-134. Arakaki, A (2015a). La pobreza por ingresos en la Argentina en el largo plazo. Realidad Económica, (289), 85107. http://www.iade.org.ar/system/files/ediciones/re289.pdf Arakaki, A. (2015b). Trabajadores precarios del sector privado. Una evaluación de su volumen, composición y remuneración en la experiencia argentina reciente. En J. Lindenboim y A. Salvia (Coords.), Hora de balance: proceso de acumulación, mercado de trabajo y bienestar: Argentina, 2002-2014 (pp. 173-210). Eudeba. Arceo, N., Monsalvo, A., Schorr, M. y Wainer, A. (2008). Empleo y salarios en Argentina: una visión de largo plazo. Capital Intelectual.

Beccaria, L. (2007). El mercado de trabajo luego de la crisis. Avances y desafíos. En B. Kosacoff (Ed.), Crisis, recuperación y nuevos dilemas. La economía argentina 2002-2007 (pp. 357-394). CEPAL.

Beccaria, L. y Groissman, F. (2015). Informalidad y segmentación del mercado laboral: el caso de Argentina. Revista CEPAL, (117), 127-143. https://www.cepal.org/sites/default/files/publication/files/39471/REV117_Beccaria-Groisman.pdf Beccaria, L. y Maurizio, R. (2017). Mercado de trabajo y desigualdad en la Argentina. Un balance de las últimas tres décadas. Sociedad, (37), 15-41.

https://publicaciones.sociales.uba.ar/index.php/revistasociedad/article/view/2971/2460

Braslavsky, C. (1989). La discriminación educativa. Miño y Dávila Editores.

Brusilovsky, S. (1995). Educación de adultos: conceptos, realidades y propuestas. Diálogos, 1,38-44.

Brusilovsky, S. y Cabrera, M. E. (2006). La normativa para educación de adultos. Una de las claves para entender la vida escolar. En S. Brusilovsky, Educación escolar de adultos. Una identidad en construcción (pp. 69-79). NOVEDUC.

Burachik, G. (2012). La crisis del capitalismo en Argentina desde los años '70. Hic Rhodus. Crisis capitalista, polémica, controversias, (3), 11-40. https://publicaciones.sociales.uba.ar/index.php/hicrhodus/article/view/955/843

Burgos, A. (2015). Jóvenes, educación y trabajo. Acerca del Plan FinEs como una estrategia de inclusión social y laboral. $12^{\circ}$ Congreso Nacional de Estudios del Trabajo. Facultad de Ciencias Económicas de la UBA, Buenos Aires. https://www.aset.org.ar/2015/ponencias/7_Burgos.pdf Cazón, F., Kennedy, D. y Lastra, F. (2016). Las condiciones de reproducción de fuerza de trabajo como forma de la especificidad de la acumulación de capital en Argentina: evidencias concretas desde mediados de los 
'70. Trabajo y Sociedad, (27), 305-327.

https://www.unse.edu.ar/trabajoysociedad/27\%20CAZON\%20KENNEDY\%20LASTRA\%20fuerza\%20de\%20tr abajo\%20superpoblacion\%20relativa.pdf

Coatz, D. y Schteingart, D. (2016). La industria argentina en e/ siglo XXI: entre los avatares de la coyuntura y los desafíos estructurales. Techint. https://www.uia.org.ar/centro-de-estudios/2605/la-industria-argentinaen-el-siglo-xxi-entre-los-avatares-de-la-coyuntura-y-los-desafios-estructurales/

Cruces, G. y Gasparini, L. (2009). Desigualdad en Argentina. Una revisión de la evidencia empírica. Segunda Parte. Desarrollo Económico, 49(193), 3-29. https://www.jstor.org/stable/20627860

De la Fare, M. (2013). Estudiantes del nivel secundario de la Educación Permanente de Jóvenes y Adultos (EPJA). ME-DINIECE.

Donaire, R. (2007). ¿Quiénes son los "trabajadores por cuenta propia"? (Argentina, 1980-2001). Lavboratorio,

(20), 58-64.

Donaire, R. (2019). Superpoblación relativa en Argentina. Un análisis a partir de tres mediciones

(2003/2010/2017). 14 Congreso Nacional de Estudios del Trabajo. Facultad de Ciencias Económicas de la UBA, Buenos Aires. https://aset.org.ar/2019/ponencias/4_Donaire.pdf

Donaire, R., Rosati, G., Cavalleri, S. y Mattera, P. (2017). Superpoblación relativa en Argentina. Construcción de un instrumento para su relevamiento sistemático y estandarizado. PIMSA.

Espro, M. y Zorattini, D. (2012). La miseria de las PYMES. Pobreza y desarrollo en la Argentina reciente. $V$ Jornadas de Economía Crítica. Facultad de Ciencias Económicas de la UBA, Buenos Aires.

https://www.academia.edu/40077114/La_miseria_de_las_Pymes_MEyDZ

Falappa, F. y Andrenacci, L. (2009). La política social de la Argentina democrática (1983-2008). Universidad

Nacional de General Sarmiento.

González, F. (2017). El empleo y el trabajo como dimensiones de análisis: El proceso de inserción laboral de estudiantes del FinEs2. Questión, 7(56). https://doi.org/10.24215/16696581e011

Graña, J. M. (2012). Las condiciones productivas de las empresas como causa de la evolución de las condiciones de empleo. [Tesis de doctorado]. Universidad de Buenos Aires. Biblioteca Digital de la Facultad de Ciencias Económicas de la UBA.

http://bibliotecadigital.econ.uba.ar/econ/collection/tesis/document/1501-1221_GranajM

Grassi, E. y Alayón, N. (2005). Condiciones de empleo y pobreza en la Argentina. Las consecuencias de la política neoliberal de los años 90. Revista de la Facultad de Ciencias Económicas de la UNMSM, (26), 111-128. https://sisbib.unmsm.edu.pe/bibvirtualdata/publicaciones/economia/26/a07.pdf

Hirsch, D. (2020). Reformas de la educación técnica y transformaciones productivas: el vínculo entre el Estado, las empresas y las escuelas para la formación de la fuerza de trabajo (1992-2014) [Tesis de doctorado no publicada]. Universidad de Buenos Aires.

Ibánez Martín, M. (2017). Inclusión y equidad. Un análisis con base en el acceso y logros para el nivel medio de educación en Argentina. Semestre económico, 20(43), 111-138. https://doi.org/10.22395/seec.v20n43a5 Iñigo Carrera, J. (2007). La formación económica de la sociedad argentina. Imago Mundi. Iñigo Carrera, J. (2005). La fragmentación internacional de la subjetividad productiva de la clase obrera. 70 Congreso Nacional de Estudios del Trabajo. Facultad de Ciencias Económicas de la UBA, Buenos Aires. https://www.aset.org.ar/congresos/7/12032.pdf

Iñigo, L. y Río, V. (2017). Extensión de la escolaridad y obligatoriedad de la escuela secundaria en Argentina: el papel de la universalización de la lectura y escritura. Universitas humanística, 83, 206-236.

http://dx.doi.org/10.11144//Javeriana.uh83.eeoe 
Kennedy, D. (2014). Producción y apropiación de valor en Argentina: el rol del deprimido salario real.

Problemas del desarrollo, 176(45), 157-182. http://www.scielo.org.mx/pdf/prode/v45n176/v45n176a8.pdf

Kennedy, D. y Graña, J. M. (2010). El empobrecimiento de los trabajadores como fuente de excedente en economías con débil dinámica productiva. Argentina desde mediados del siglo XX. Pecvnia, (10), 231-263. http://revpubli.unileon.es/ojs/index.php/Pecvnia/article/view/652/574

Kornblihtt, J., Seiffer, T. y Villanova, N. (2014). De la caída relativa a la caída absoluta del salario real en la Argentina (1950-2013). Revista Científica Guillermo de Ockham, 12(2), 41-50.

https://www.redalyc.org/articulo.oa?id $=105338606005$

Lavopa, A. (2005). Heterogeneidad estructural y segmentación del mercado de trabajo. Evidencias para el caso argentino durante el periodo 1991-2004. 70 Congreso Nacional de Estudios del Trabajo. Facultad de Ciencias Económicas de la UBA, Buenos Aires. https://www.aset.org.ar/congresos/7/06004.pdf Lindenboim, J. (2001). La (des)protección laboral en Argentina. El mercado de trabajo urbano en los noventa. Latin American Studies Association.

Marticorena, C. (2005). Precariedad laboral y caída salarial. El mercado de trabajo en la Argentina Post Convertibilidad. $7^{\circ}$ Congreso Nacional de Estudios del Trabajo. Buenos Aires, Argentina.

https://www.aset.org.ar/congresos/7/02008.pdf

Mendonça, M. y Pérez Trento, N. (2020). El devenir del sistema universitario público en la Argentina a través de sus olas expansivas: Diferenciación en la formación de fuerza de trabajo y acumulación de capital. (19712015). Archivos de Políticas Educativas, 28(49). https://doi.org/10.14507/epaa.28.4914 Montesinos, M., Schoo, S. y Sinisi, L. (2010). Aportes para pensar la Educación de Jóvenes y Adultos en el nivel secundario. Un estudio desde la perspectiva de los sujetos. ME-DINIECE.

Pecarrere, F. (2014). Plan FinEs 2: Algunas experiencias en la ciudad de La Plata [Tesis de grado]. Universidad Nacional de La Plata. Repositorio institucional de la Facultad de Humanidades y Ciencias de la Educación de la Universidad Nacional de La Plata. http://www.memoria.fahce.unlp.edu.ar/tesis/te.1095/te.1095.pdf Poy, S. y Salvia, A. (2017). Transformaciones económicas, reformas estructurales y desigualdad distributiva del ingreso monetario familiar en la Argentina (1974-2014). V Seminario Internacional Desigualdad y Movilidad en América Latina, Buenos Aires. http://www.memoria.fahce.unlp.edu.ar/trab_eventos/ev.10651/ev.10651.pdf Riquelme, G., Herger, N. y Sassera, J. (2018). Deuda social educativa con jóvenes y adultos. Entre el derecho a la educación, los discursos de las políticas y las contradicciones de la inclusión y la exclusión. Editorial de la Facultad de Filosofía y Letras, UBA.

Rodríguez, L. (2008). Situación presente de la educación de personas jóvenes y adultas en Argentina. CEAALCREFAL.

Rodríguez, L. (2009). Educación de adultos en la historia reciente de América Latina y el Caribe. Revista Electrónica de Educación y Formación Continua de Adultos, 3(1), 64-82.

http://campus.usal.es/ efora/efora_03/monografico_efora_03.pdf

Salvia, A. (2010). Heterogeneidad estructural y segmentación del mercado de trabajo en dos contextos económicos. Argentina 1998-2006. VI Congreso de la Asociación Latinoamericana de Sociología del Trabajo, Distrito Federal. https://www.aacademica.org/agustin.salvia/80 Seiffer T. y Rivas, G. (2017). La política social como forma de reproducción de la especificidad histórica de la acumulación de capital en Argentina (2003-2016). Estudios del trabajo, (54), 91-117. https://aset.org.ar/ojs/revista/article/view/4 
Seiffer, T. (2013). Bases de la asistencialización de la Política Social en Argentina (1980-2010). Documentos de Trabajo Social, (51), 69-88. https://core.ac.uk/download/pdf/159292428.pdf

Sirvent, T. (2005). Los jóvenes y adultos en la Argentina en el contexto de las políticas de neoconservadurismo. Pensamiento Educativo, (37), 277-295.

http://redae.uc.cl/index.php/pel/article/view/26821/21557

Sucunza, M. (2016). La incidencia del Plan Fines II en las trayectorias educativo-laborales de las personas que participaron de ésta experiencia. Revista Oficio, (2), 4-61. http://revistaoficio.org/wp-

content/uploads/2016/07/Oficio-N2-Completa.pdf

Tiramonti, G. (2009). Una aproximación a la dinámica de la fragmentación del sistema educativo argentino. Especificaciones teóricas y empíricas. En N. Montes y G. Tiramonti (Comps.), La escuela media en debate. Problemas actuales y perspectivas desde la investigación (pp. 25-38). Manantial/FLACSO.

Tiramonti, G. (2019). La escuela media argentina: el devenir de una crisis. Propuesta Educativa, (51), 78-92. http://propuestaeducativa.flacso.org.ar/wp-content/uploads/2019/11/PropuestaEducativa51-articuloTIRAMONTI.pdf

Topasso, P., Castañeda, J. y Ferri, P. (2015). La Demanda Potencial por Educación de Jóvenes y Adultos, de acuerdo al procesamiento de datos del Censo Nacional de Población, Hogares y Viviendas 2010. IX Jornadas de Investigación en Educación. Facultad de Filosofía y Humanidades de la UNC, Córdoba.

Viego, V. (2015). Políticas públicas para la terminalidad educativa: el caso de Plan Fines en Argentina. Archivos Analíticos de Políticas Educativas, 23(116). http://dx.doi.org/10.14507/epaa.v23.1929 Villarreal, J. (1985). Los hilos sociales del poder. En E. Jozami, P. Paz y J. Villarreal (Eds.), Crisis de la dictadura argentina. Política económica y cambio social (pp. 201-281). Siglo XXI.

Zack, G., Schteingart, D. y Favata, F. (2017). Pobreza e indigencia en Argentina (2003-2017): construcción de una serie complete y metodológicamente homogénea. L/l Reunión Anual de la Asociación Argentina de Economía Política. Universidad Nacional de Río Negro, Río Negro.

https://aaep.org.ar/anales/works/works2017/zack_schteingart.pdf 